\title{
Tumor suppressor PDCD4 modulates miR-184- mediated direct suppression of C-MYC and BCL2 blocking cell growth and survival in nasopharyngeal carcinoma
}

\author{
Yan Zhen ${ }^{1,2,3,8}$, Zhen Liu ${ }^{*, 1,2,8}$, Huiling Yang ${ }^{2,4,8}$, Xiaoli Yu ${ }^{2,8}$, Qiangyun $\mathrm{Wu}^{2}$, Shengni Hua ${ }^{2}$, Xiaobin Long ${ }^{2,5}$, Qingping Jiang ${ }^{2,6}$, \\ Ye Song ${ }^{2,7}$, Chao Cheng ${ }^{2,5}$, Hao Wang ${ }^{2}$, Menyang Zhao ${ }^{2}$, Qiaofen $\mathrm{Fu}^{2}$, Xiaoming Lyu ${ }^{2}$, Yiyu Chen ${ }^{2}$, Yue Fan ${ }^{2}$, Yan Liü ${ }^{2}$, Xin Li, ${ }^{, 2}$ \\ and Weiyi Fang ${ }^{\star 2}$
}

Programmed cell death 4 (PDCD4), a novel tumor suppressor, inhibits cell proliferation, migration and invasion as well as promotes cell apoptosis in tumors. However, the molecular mechanism of its tumor-suppressive function remains largely unknown in tumors including nasopharyngeal carcinoma (NPC). In this study, downregulated PDCD4 expression was significantly associated with the status of NPC progression and poor prognosis. PDCD4 markedly suppressed the ability of cell proliferation and cell survival by modulating C-MYC-controlled cell cycle and BCL-2-mediated mitochondrion apoptosis resistance signals, and oncogenic transcription factor C-JUN in NPC. Furthermore, miR-184, a tumor-suppressive miRNA modulated by PDCD4 directly targeting BCL2 and C-MYC, participated in PDCD4-mediated suppression of cell proliferation and survival in NPC. Further, we found that PDCD4 decreased the binding of C-Jun to the AP-1 element on the miR-184 promoter regions by PI3K/AKT/JNK/C-Jun pathway and stimulated miR-184 expression. In clinical fresh specimens, reduced PDCD4 mRNA level was positively correlated with miR-184 expression in NPC. Our studies are the first to demonstrate that PDCD4 as tumor suppressor regulated miR-184-mediated direct targeting of BCL2 and C-MYC via PI3K/AKT and JNK/C-Jun pathway attenuating cell proliferation and survival in NPC.

Cell Death and Disease (2013) 4, e872; doi:10.1038/cddis.2013.376; published online 24 October 2013

Subject Category: Cancer

Programmed cell death 4 (PDCD4) is localized to chromosome band 10q24 and comprises two basic domains at the $\mathrm{N}$-terminus and $\mathrm{C}$-terminus involving nuclear localization signals and two conserved helical MA-3 domains associated with protein-protein interactions in elF4GI and elF4GII with the ATP-dependent RNA helicase elF4A proposing the repression of translation initiation. ${ }^{1,2}$ During investigation of apoptosis, PDCD4 was first identified as an upregulated gene. However, a growing number of reports have pointed to PDCD4 as a new tumor suppressor gene involved in the apoptotic machinery, which suppresses cell transformation, tumorigenesis and invasion at present. $^{3-6}$ Furthermore, PDCD4 expression is significantly downregulated in various human cancers, as well as in cancer cell lines, and this has been associated with a poor patient prognosis., ${ }^{4,8}$ However, the detailed functional mechanism of PDCD4 in tumor pathogenesis is not clear yet, including NPC.
NPC is the most common cancer in certain regions of Southern China and Southest Asia than elsewhere, with viral, dietary and genetic factors implicated in its causation, which may often drive abnormal gene expression and contribute to the initiation and development of NPC. ${ }^{9-14}$ In previous investigation, we used cDNA microarray to examine differentially expressed genes among NPC tissues and nasopharynx tissues. By means of the analysis of BRB array tools, the expression of PDCD4 was shown to be markedly decreased in NPC tissues, suggesting a possible role of PDCD4 in suppressing the pathogenesis of NPC. ${ }^{9}$ In this study, we investigated the expression pattern, the effects and molecular basis of PDCD4 on cell growth and apoptosis in NPC. Our studies demonstrated that reduced PDCD4 expression as a tumor suppressor modulated miR-184 stimulating NPC progression and poor prognosis.

\footnotetext{
${ }^{1}$ Department of Pathology, Basic School, Guangzhou Medical University, Guangzhou 510182, PR China; ${ }^{2}$ Cancer Research Institute, Southern Medical University, Guangzhou 510515, PR China; ${ }^{3}$ nstitute of Respiratory Diseases, Affiliated Hospital of Guangdong Medical College, Zhanjiang 524000, PR China; ${ }^{4}$ School of Pharmacy, Guangdong Medical College, Dongguan 523808, PR China; ${ }^{5}$ Otorhinolaryngology of Zhujiang Hospital, Southern Medical University, Guangzhou 510282, PR China; ${ }^{6}$ Department of Pathology, Third Affiliated Hospital of Guangzhou Medical College, Guangzhou 510150, PR China and ${ }^{7}$ Department of Neurosurgery, Nanfang Hospital, Southern Medical University, Guangzhou 501515, PR China

${ }^{*}$ Corresponding authors: Z Liu or X Li or W Fang, Oncology, Cancer Research Institute, Southern Medical University, Tonghe Road, Guangzhou, Guangdong Province 510515, PR China. Tel: +86 0206278 9438; Fax: +86 0206164 8226; E-mails: fangweiyi1975@163.com or xinli268@gmail.com or narcissus_jane@163.com

${ }^{8}$ These authors contributed equally to this work.

Keywords: PDCD4; NPC; miR-184; BCL2; C-MYC

Abbreviations: NPC, nasopharyngeal carcinoma; PDCD4, programmed cell death 4; NP, nasopharyngeal tissues; PI3K, phosphoinositide 3-kinase; AKT, v-akt murine thymoma viral oncogene homolog 1; JNK, C-Jun N-terminal kinase 1; C-Jun, jun proto-oncogene; BCL2, B-cell CLL/lymphoma 2; ACTB, actin beta; qRT-PCR, real-time quantitative reverse transcription PCR; MTT, 3-(4,5-dimethylthiazol-2-yl)-2,5-diphenyltetrazolium bromide; cDNA, complementary DNA; miRNA, microRNA

Received 21.6.13; revised 23.8.13; accepted 26.8.13; Edited by E Candi
} 


\section{Results}

PDCD4 is lowly expressed in NPC. From Lee et al.'s ${ }^{15}$ microarray data (GSE2370), PDCD4 was downexpressed in NPC tissues compared with NP tissues by an average of 0.60 folds (Figure 1A). This expression pattern was similar to our microarray data comparing NPC cells and NP tissues. ${ }^{9}$ QPCR analysis indicated that the PDCD4 expression level was significantly decreased in eight NPC cell lines and NPCs in comparison to NP tissues $(P<0.0001)$ (Figure 1B). Furthermore, we also observed the decreased expression tendency of PDCD4 in clinical stage of III-IV compared with I-II (Supplementary Figure S1A) and lymph node metastasis (N classification) of N2-N3 compared with N0-N1 (Supplementary Figure S1B). However, no statistically significant difference was shown in T3-T4 compared with T1-T2 (Supplementary Figure S1C) as well as in M1 compared with M0 (Supplementary Figure S1D). Similar to mRNA expression pattern, the PDCD4 protein expression was significantly decreased in five NPCs in comparison to three NPs using western blot examination (Figure 1C). Finally, immunohistochemical staining indicated that PDCD4 protein was lowly expressed in NPC samples compared with noncancerous tissues $(P<0.001$, Figure 1D; Supplementary Table S9).

Downregulated protein expression of PDCD4 was associated with NPC progression and poor prognosis. We analyzed the relationship between clinicopathologic characteristics and PDCD4 expression levels in individuals with NPC (Supplementary Table S10). We did not find a significant association of PDCD4 expression levels with patient's age, sex, smoking status, family tumor history, disease recurrence or distant metastasis (M classification) in 190 NPC cases. However, we observed that the reduced expression level of PDCD4 was markedly correlated with tumor size (T classification; $P=0.026$ ), lymph node metastasis (N classification; N0-N1 versus N2-N3, $P=0.036$ ) and clinical stage (I-II versus III-IV, $P=0.002$ ) in NPC patients. In addition, we observed that the level of PDCD4 protein expression was significantly correlated with overall survival, as patients with lower levels of PDCD4 expression had poorer survival (Figure 1E) than those with higher levels of PDCD4 expression $(P<0.001)$. Furthermore, we also found that increased expression of PDCD4 showed better prognosis in NPC patients, regardless of disease stage, T, N and M classifications (Supplementary Figures S2A-F). Multivariate analysis showed that decreased PDCD4 expression was a poor independent prognostic factor for NPC patients $(P<0.001$; Supplementary Table S11).

PDCD4 inhibits cell proliferation, cell cycle and induces cell apoptosis. To study the biological functions of PDCD4, we used a PDCD4-less-expressing NPC cell line 5-8F and introduced PDCD4 into these cells using lentivirus containing PDCD4 gene. Clone1 and 2 cells with the highest PDCD4 expression were obtained in seven stably transfected cell clones by qPCR and western blot assays (Figures 2a and b). The growth curves and colony formation assay showed that PDCD4 significantly inhibited cell proliferation of clone1 and
2 cells in comparison to scramble and parental line $5-8 \mathrm{~F}$ cells (Figures 2c and d). Furthermore, we also observed that overexpressed PDCD4 blocked cell cycle transition from G1 to $S$ and $G 2$ phase(Figure 2e) and induced cell apoptosis in clone1 and 2 cells compared with their control cells(Figure 2f). To further confirm the growth-suppressive effect of PDCD4, we performed in vivo tumorigenesis study in nude mice. The results showed that the average volume of these two clone cells was significantly decreased compared with scramble cells (Figure 2g). Immunohistochemistry examination indicated increased PDCD4 expression in clone 1 and 2 xenograft tumor specimens than in scramble cells (Figure 2h). Interestingly, inversed results were also observed in short hairpin RNA (shRNA)-mediated suppression of PDCD4 in HONE1 and SUNE1 NPC cells with the highest mRNA expression levels of PDCD4 (Supplementary Figures $\mathrm{S} 3 \mathrm{~A}-\mathrm{C}$ ). We found that suppressing PDCD4 significantly elevated cell proliferation, colony formation, cell cycle transition and cell survival in ShPDCD4 NPC cells in comparison to shScramble NPC cells (Supplementary Figures S3D-G).

PDCD4 regulated PI3K/AKT and JNK, cell cycle, cell apoptosis and C-JUN. PDCD4 has been reported to regulate PI3K/AKT pathway and JNK expression in some tumors. ${ }^{16,17}$ In this study, we also examined the effect of PDCD4 on the expression of PI3K/AKT and JNK pathways in NPC. Western blot analysis showed that overexpressed or reduced PDCD4 significantly reduced or increased the expression of phos-PI3K and phos-AKT (Figures $3 a$ and b), but not their total protein levels (Supplementary Figures S4A and $B$ ), respectively. Furthermore, we also observed the suppressed or elevated expression of JNK in PDCD4overexpressed or PDCD4-reduced NPC cells, respectively (Figures 3a and b). In previous studies, C-JUN, cell cycle and cell apoptosis signals-associated moleculars as downstream signals had been shown to be modulated by PI3K/AKT and JNK pathways, ${ }^{8,11,18}$ we thus examined their protein expression in PDCD4-overexpressed NPC 5-8F cells and PDCD4-suppressed NPC HONE1 and SUNE1 cells. Using western blotting analysis, we first examined the expression of C-JUN (AP1) and cell cycle factors associated with cell proliferation. We found that knocking down endogenous PDCD4 expression or introduction of PDCD4 induced or blocked the expression of C-JUN, pRB C-MYC, CCND1 and E2F1, respectively (Figures $3 c$ and d). However, the change in the expression of CDK4, p15 and p27 (Supplementary Figures S4A and B) was not observed in both the cell types. Moreover, we observed that knocking down endogenous PDCD4 expression or introduction of PDCD4 induced or decreased the expression of BCL2-mediated suppression of the cleavage of CASP9, 3, 6, 7, and PARP in NPC cells (Figures $4 a$ and b).

PDCD4 modulated the expression of miR-184 in NPC. To investigate the effect of PDCD4 on miRNAs in NPC, we used miRNA chip to compare the differential miRNAs between shPDCD4 and shScramble SUNE1 cells. We found that miR184 was markedly downregulated in shPDCD4 cells (Figure 5a). Further, we used qPCR to confirm the reliability 


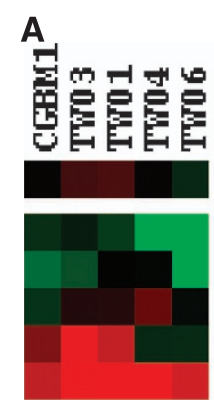

\section{B}

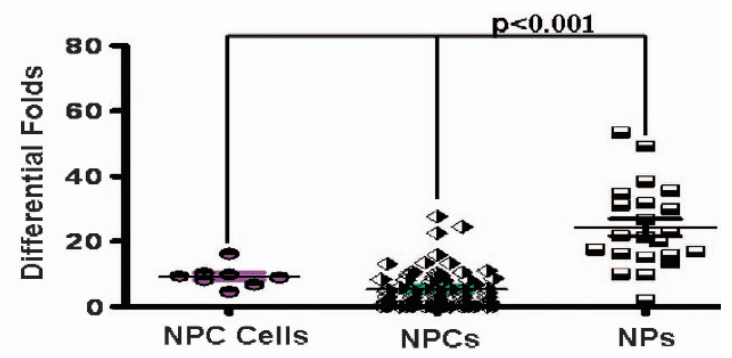

C

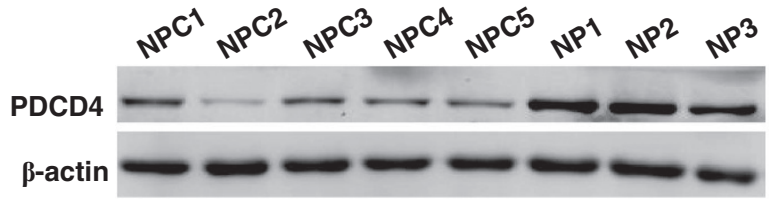

D
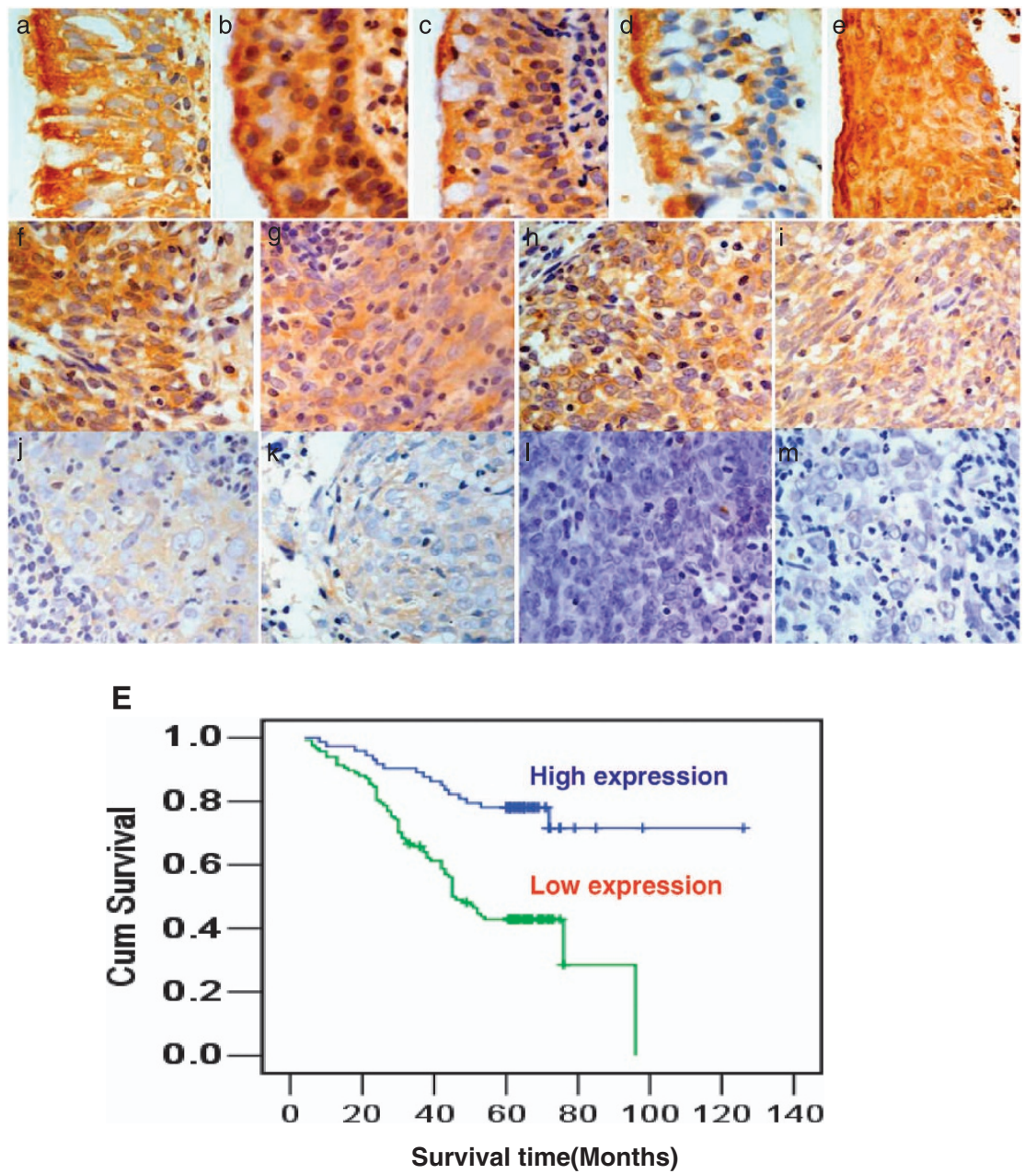

Figure 1 Reduced expression of PDCD4 promoted the progression and poor prognosis of NPC patients. (A) Decreased PDCD4 expression was shown in NPC by microarray data analysis of GSE2370 data set retrieved from the GEO database. (B) Compared with nasopharynx tissues, PDCD4 expression was markedly decreased in NPC tissues and NPC cells $(P<0.001)$. (C) Compared with nasopharynx tissues, PDCD4 protein expression was significantly downregulated in NPC tissues. (D) PDCD4 expression was progressively decreased in NPC samples compared with normal nasopharynx tissues, squamous epithelium and atypical hyperplasia. (1) PDCD4 expression in normal epithelium, a-d: strong expression; (2) PDCD4 expression in squamous epithelium, e: strong expression; (3) PDCD4 expression in NPC, f-i: Strong and positive expression; j-m: weak expression and negative expression. (E) Lower levels of PDCD4 reduced the survival time of NPC patients 

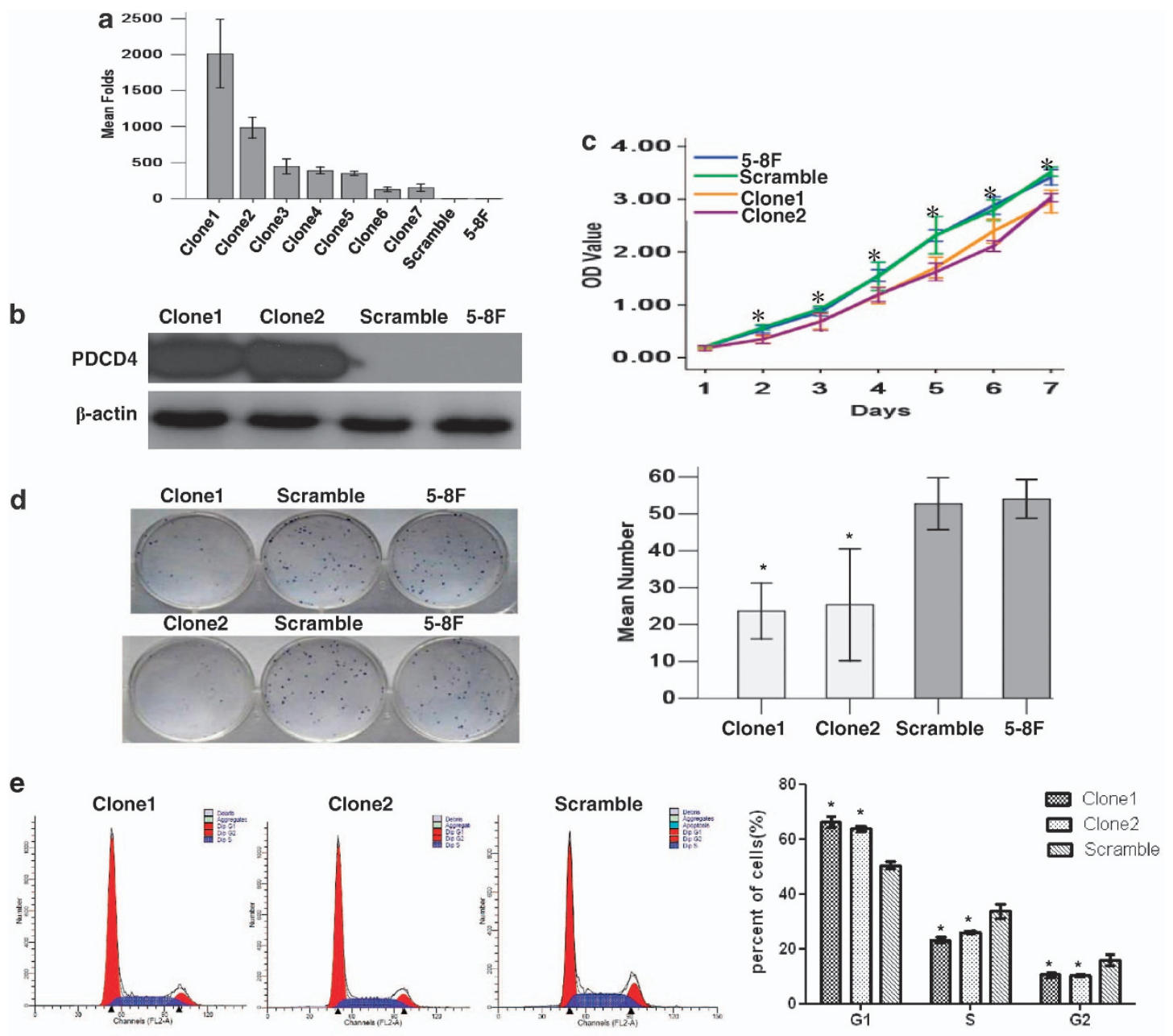

f
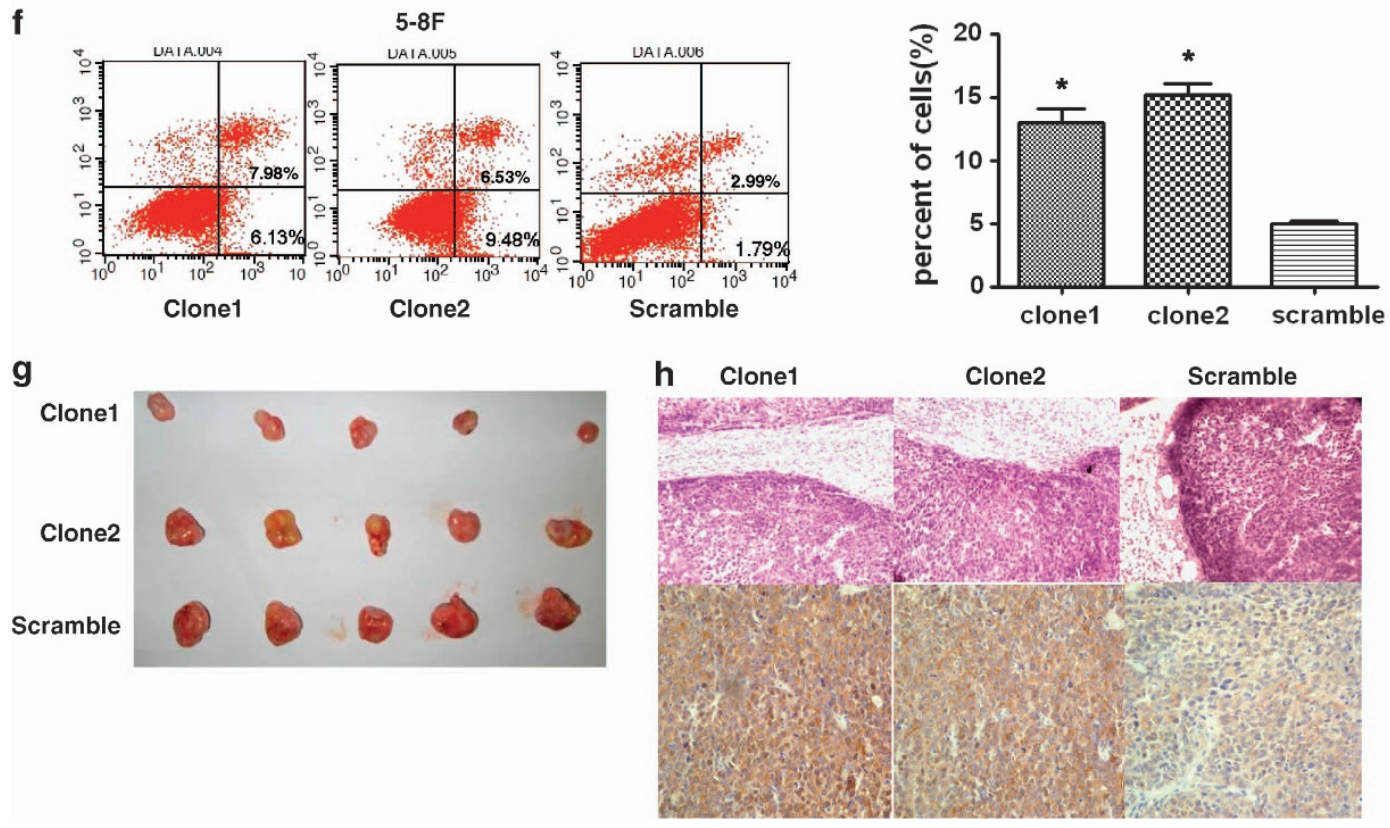
a

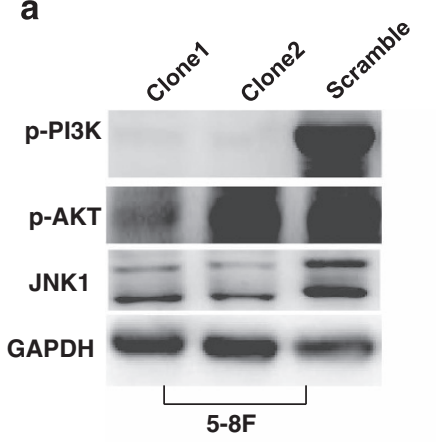

C

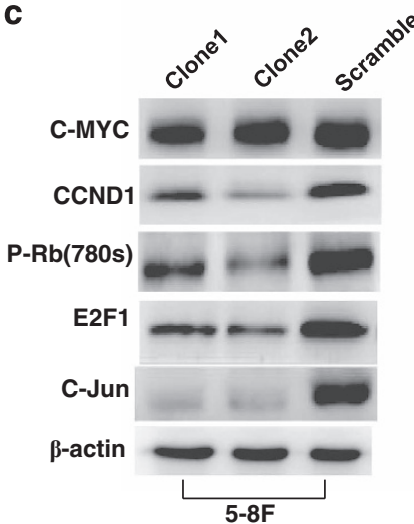

b

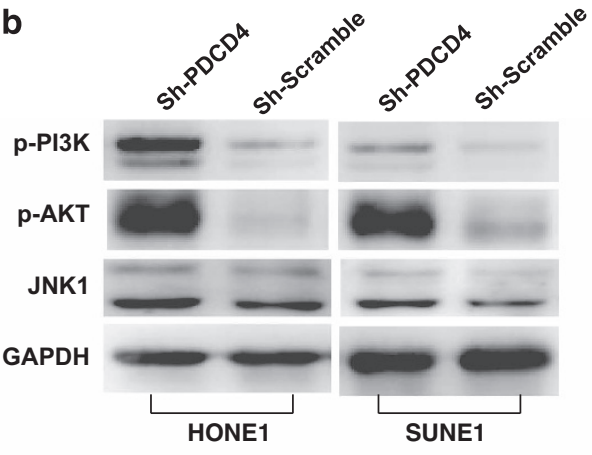

d

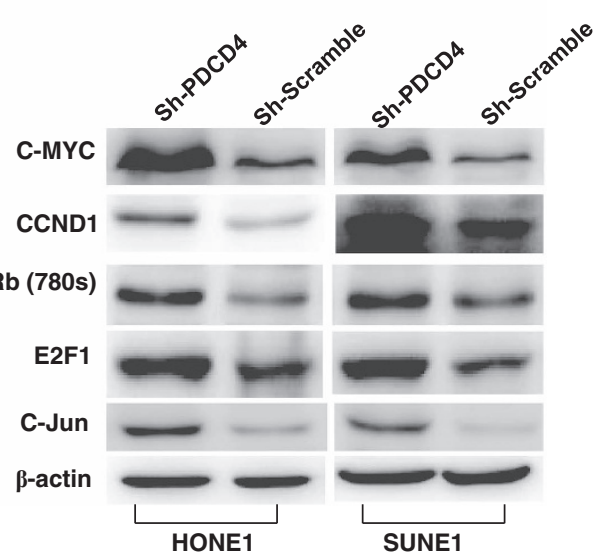

Figure 3 PDCD4 controls the expression of cell cycle and transcription factor C-JUN in NPC via PI3K/AKT/JNK pathway. (a) Overexpressed PDCD4 suppressed the expression of pPI3K, pAKT and JNK in clone 1 and 2 cells compared with scramble cells. (b) Reduced PDCD4 elevated the expression of pPI3K, pAKT and JNK in shPDCD4 SUNE1 and HONE1 cells compared with their respective controls. (c) Restored PDCD4 inhibited the expression of C-MYC, CCND1, pRB (Ser780), E2F1 and C-JUN in clone 1 and 2 cells compared with scramble cells. (d) Downregulated PDCD4 expression stimulated the C-MYC, CCND1, pRB (Ser780), E2F1 and C-JUN expression in shPDCD4 SUNE1 and HONE1 cells compared with their respective controls
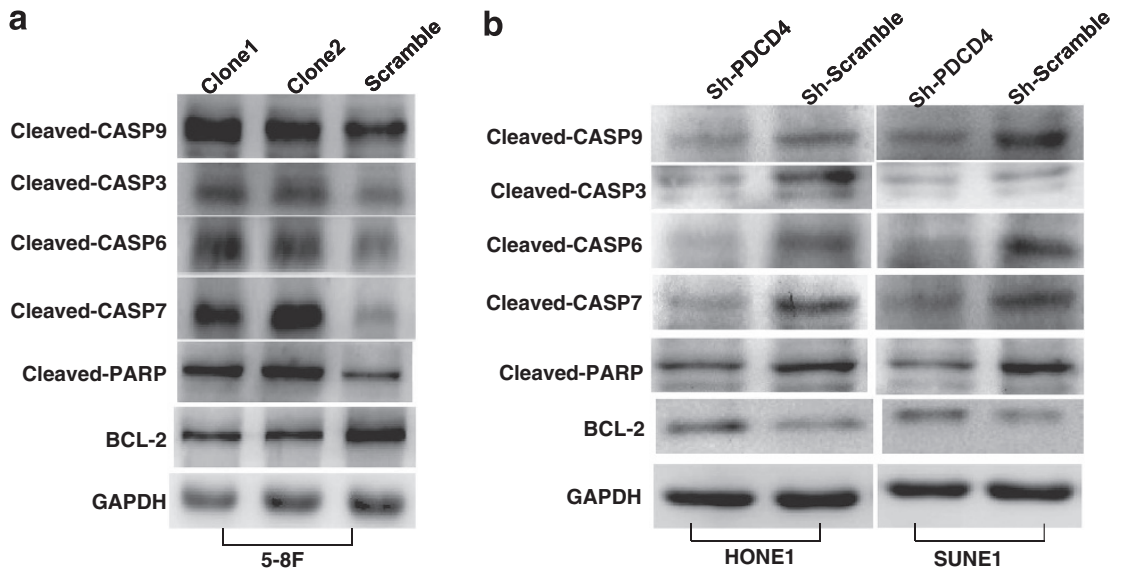

Figure 4 PDCD4 mediated cell apoptosis in NPC. (a) Restoration of PDCD4 expression induced the cleavage of CASP9, 3, 6, 7 and PARP and suppressed BCL2 expression. (b) Stably knocking down the expression of PDCD4 by shRNA reduced the cleavage of CASP9, 3, 6, 7 and PARP and elevated the expression of BCL2

Figure 2 Overexpressed PDCD4 expression suppressed cell proliferation and cell cycle transition and induced cell apoptosis. (a) PDCD4 was highly reexpressed in clone 1-7 detected by qPCR after infection and selection. (b) Restoration of PDCD4 protein expression was examined in clone 1 and 2 single clone cells compared with scramble and 5-8F cells by western blot. Because of the fusion protein from PDCD4 and green fluorescent protein (GFP), the fusion protein of scramble and 5-8F cells cannot be detected. (c, d) In vitro proliferative ability of NPC cells was significantly inhibited in PDCD4-overexpressing clone 1 and 2 cells compared with scramble and 5-8F cells by MTT assay and plate clone assay. (e) Restored PDCD4 expression markedly inhibited the cell cycle transition from G1 to S and G2 in vitro. (f) Increased PDCD4 expression induced cell apoptosis in NPC 5-8F cells. (g) Compared with scramble cells, tumorigenicity of clone 1 and 2 cells was markedly reduced in vivo. (h) PDCD4 protein was highly expressed in PDCD4-overexpressing clone 1 and 2 cells compared with scramble cells by immunohistochemistry assay. Data are presented as mean \pm S.D. of three independent experiments $\left({ }^{*} P<0.05\right)$ 

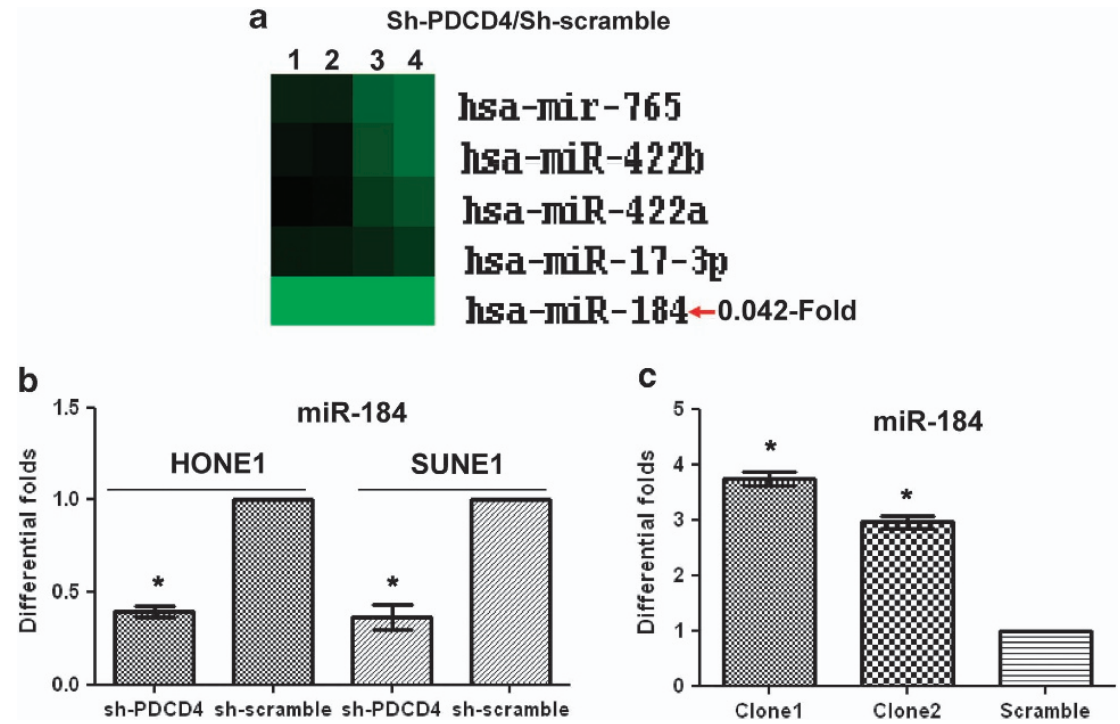

Figure 5 PDCD4 regulated the expression of miR-184 in NPC. (a) miR-184 was notably downregulated in shRNA-PDCD4-treated SUNE1 NPC cells by miRNA array analysis. (b) Real-time PCR confirmed the downregulated expression of miR-184 in shPDCD4-treated SUNE1 and HONE1 cells. (c) Upregulated expression of miR-184 was shown in PDCD4-overexpressed NPC 5-8F cells

of miRNA chip in knocking down the expression of PDCD4 in NPC cells (Figure 5b). Furthermore, we also validated the upregulated expression of miR-184 in PDCD4-overexpressed 5-8F NPC cells (Figure 5c).

Overexpressed miR-184 inhibits cell proliferation and induces apoptosis in NPC. To study the biological functions of miR-184, we introduced miR-184 mimics into NPC SUNE1 and 5-8F cell lines. Compared with negative control of miR-184, we found that miR-184 inhibited cell growth in NPC cells by MTT assays (Supplementary Figure S5A). Furthermore, we observed that apoptotic cells were significantly increased in miR-184 mimics-treated cells (Supplementary Figure S5B). To confirm whether miR-184 can revert the promotion of cell growth in PDCD4-absent cells, we transfected miR-184 mimics into shPDCD4 HONE1 and SUNE1 cells. The results showed that miR-184 significantly decreased the cell growth in shPDCD4 NPC cells (Supplementary Figure S5C), suggesting miR-184 as a positive modulator of PDCD4 in NPC.

miR-184 directly targeting BCL2 and C-MYC suppressed cell cycle signal and stimulated mitochondrial apoptosis pathway. To study the mechanism of miR-184 suppressing cell proliferation and promoting cell apoptosis in NPC, we examined expression of cell cycle and apoptotic genes. Interestingly, similar to PDCD4-mediated pathways, miR-184 mimics suppressed the expression of pRB, C-MYC, CCND1 and E2F1 (Figure 6a) but not p15 and CDK4 in cell cycle transition (Supplementary Figure S6), downregulated the BCL2 expression and induced the cleavage of CASP9, 3, 6, 7 and PARP (Figure 6b) but not PDCD4 in apoptosis pathway (Supplementary Figure S6). Furthermore, we found that BCL2 and C-MYC were potentially direct targets of miR-184 by RNAhybrid and miRwalk assays (Supplementary Figure S7). In a preceding experiment, miR-184 mimics downregulated the protein expression of BCL2 and C-MYC in
NPC. Subsequently, we transfected miR-184 inhibitor into SUNE1 and 5-8F cells and found elevated expression levels of BCL2 and C-MYC (Figure 6c). We further performed luciferase reporter assay to determine whether miR-184 could directly target the C-MYC coding region (CDS) and $3^{\prime}$ UTR of BCL2 in NPC cells. Wild-type (wt) or mutant (mt) 3'UTR vector of BCL2 and miR-184 mimics or inhibitor NPC cells were cotransfected with NPC SUNE1 cells. The results showed a significant decrease of luciferase activity in wt vector transfected with miR-184 mimics (Figure $6 \mathrm{~d}$, lanes 1 and 2; $P<0.001$ ) or an obvious increase of luciferase activity by using miR-184 inhibitor (Figure 6d, lanes 3 and 4; $P<0.001$ ) when compared with miR control, whereas the activity of $\mathrm{mt}$ vector was unaffected (Figure $6 \mathrm{~d}$, lanes 5 and 6 , $P=0.092$ ) by using miR-184 mimics or inhibitor. Similar to the result of $\mathrm{BCL} 2$, a significant decrease of luciferase activity in wt vector of C-MYC CDS was showed by using miR-184 mimics (Figure 6e, lanes 1 and $2 P<0.001$ ) or an obvious increase of luciferase activity by transfecting miR184 inhibitor (Figure 6e, lanes 3 and 4; $P<0.001$ ) compared with $\mathrm{miR}$ control; whereas the activity of $\mathrm{mt}$ vector was unaffected (Figure 6e, lanes 5 and $6, P=0.36$ ) by using miR184 mimics or inhibitor. Taken together, these results strongly supported that BCL2 and C-MYC were the direct targets of miR-184 in NPC cells.

PDCD4 induced the expression of miR-184 by modulating PI3K/AKT/JNK/C-JUN pathway. The promoter region of human miR-184 contains AP-1 (C-JUN) binding sites. To examine the role of AP-1 (C-JUN) in regulating miR-184, we first used small-interfering RNAs (siRNAs) to suppress the expression of C-JUN in NPC SUNE1 and 5-8F cells by western blot (Figure 7a). Further, qPCR analysis indicated that the expression of miR-184 was markedly increased in both the cells with knocked down expression of C-JUN (Figure 7b), which suggested that C-JUN is an upstream regulator of miR-184. In a subsequent investigation, we used 


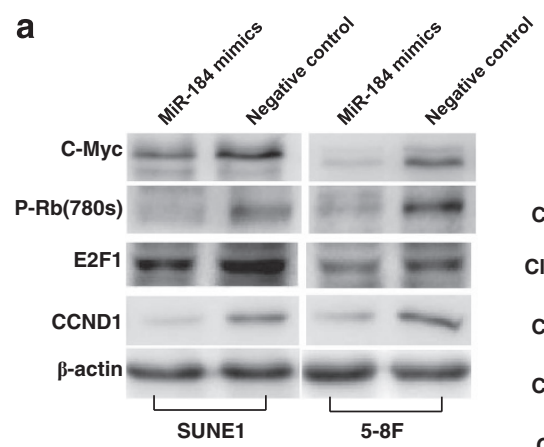

C

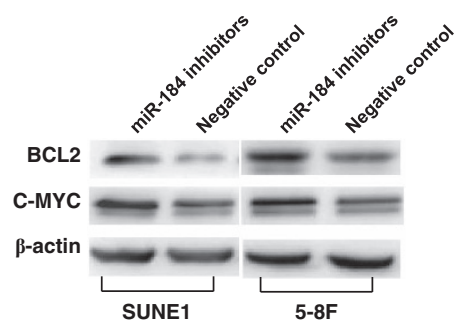

e

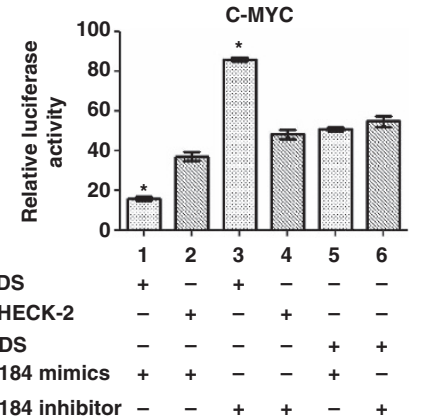

b

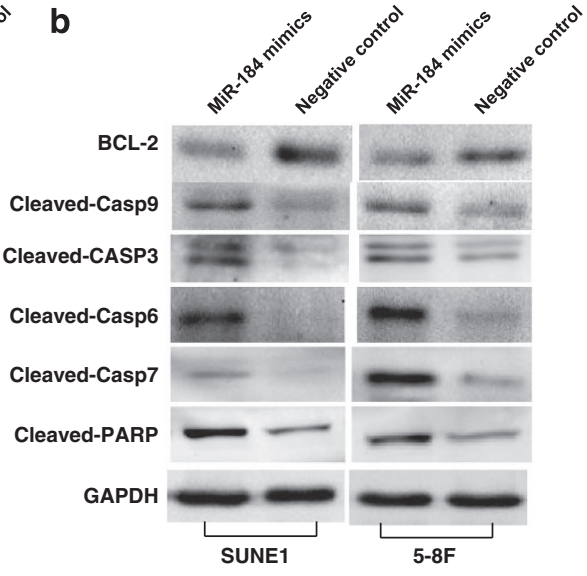

d

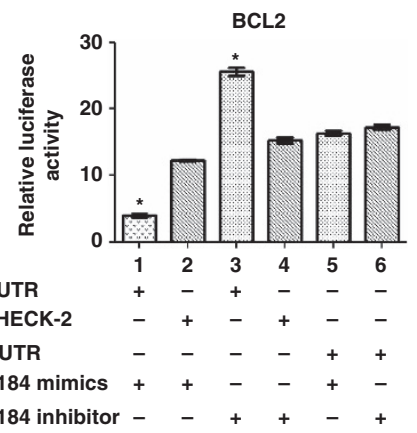

Figure 6 MiR-184 directly targeted BCL2 and C-MYC suppressing cell cycle and stimulating cell apoptosis signals. (a) The introduction of miR-184 mimics suppressed the expression of pRB (ser 780), C-MYC, CCND1 and E2F1 in cell cycle. (b) Transfection of miR-184 mimics reduced BCL2 expression and induced the cleavage of CASP9, 3, 6, 7 and PARP in apoptosis pathway. (c) BCL2 and C-MYC expression was elevated with the transfection of miR-184 inhibitor at $100 \mathrm{~nm}$ in NPC SUNE1 and 5-8F cells. (d) Luciferase reporter assay was used to determine miR-184 directly targeting the $3^{\prime} U T R$ of BCL2. (e) Luciferase reporter assay was used to determine miR-184 directly targeting the coding region of C-MYC. Data are presented as mean \pm S.D. of three independent experiments $\left({ }^{\star} P<0.05\right)$

chromatin immunoprecipitation combined with qPCR analysis to confirm that C-JUN could bind putative miR-184 promoter and activate its expression in NPC (Figure 7c). Finally, we used specific inhibitor of PI3K to suppress the expression of PI3K or specific siRNA to reduce JNK expression and observed that the protein expression of C-JUN was decreased in NPC SUNE1 and 5-8F cells (Figures $7 \mathrm{~d}$ and e). Our results demonstrated that PDCD4 stimulated the expression of miR-184 through PI3K/AKT/ JNK/C-JUN pathway.

Reduced expression tendency of miR-184 was positively correlated with the expression of PDCD4 in NPC. We further measured the mRNA levels of miR-184 in NPC specimens and NP tissues. The results showed that although the decreased tendency of average expression level of miR184 was shown in NPC specimens compared with NP tissues, the mean differences did not reach statistical significance (Supplementary Figure S8A; $P=0.24$ ). Interestingly, we observed that miR-184 expression was markedly higher in clinical I-II stage than that in clinical III-IV stage in
NPC samples (Supplementary Figure S8B; $P=0.029$ ). Furthermore, a nonsignificant increase of miR-184 expression was also observed in T1-T2 and N0-NI compared with T3-T4 and N2-N3 (Supplementary Figure S8C and D; $P=0.081$ and $P=0.085$, respectively). However, no statistically significant difference was shown in M0 compared with M1 (Supplementary Figure S8E; $P=0.677$ ). Furthermore, we found that reduced miR-184 expression was positively correlated with PDCD4 expression (Supplementary Figure $\mathrm{S} 8 \mathrm{~F} ; P=0.008)$ in the same NPC specimens.

\section{Disscussion}

PDCD4, a cytoplasmic and nuclear expression protein, has a significant role in the suppression of tumor growth. ${ }^{9}$ However, its roles and molecular mechanisms linking the initiation and development of NPC are seldom reported. ${ }^{9}$

In this study, we first observed the significantly decreased expression of PDCD4 in NPC tissues and cells compared with NP tissues by qPCR and western blot. Moreover, we observed that specific PDCD4 protein staining was found in 
a
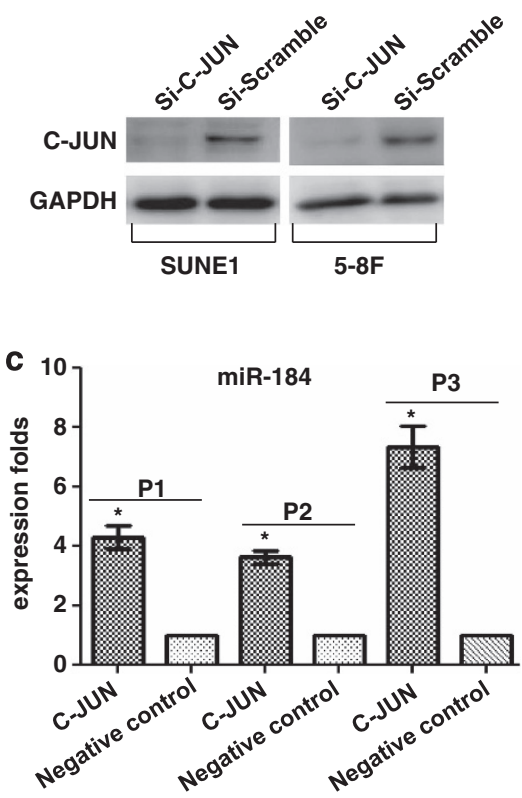

b
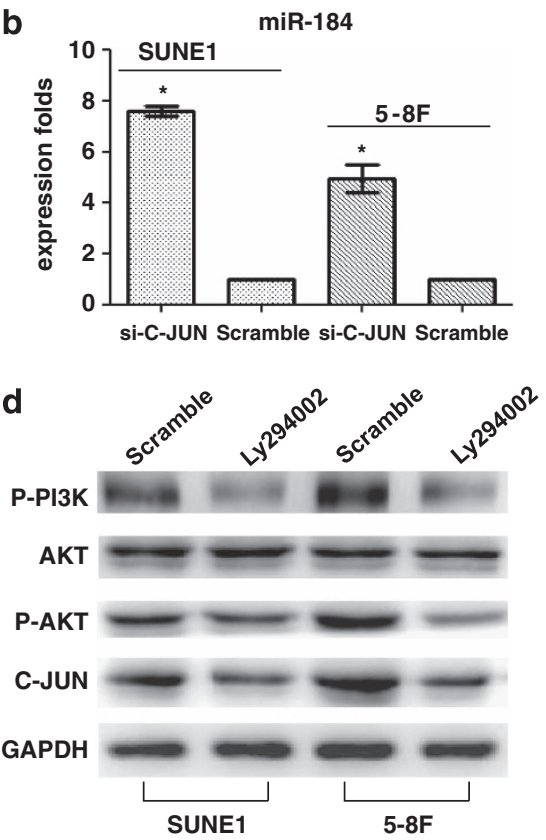

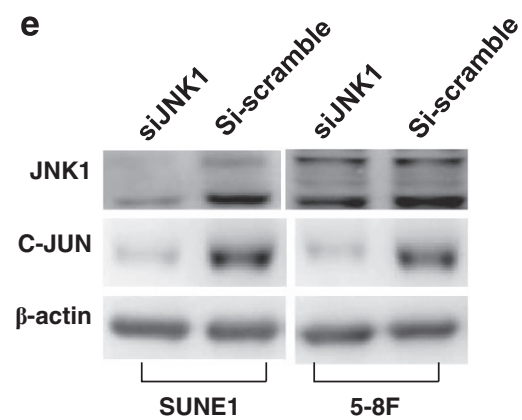

Figure 7 PDCD4 regulated the expression of miR-184 via PI3K/AKT/JNK pathway in NPC. (a) siRNAs were used to suppress the C-JUN expression by western blot examination. (b) Knocking down C-JUN expression by siRNA stimulated the expression of miR-184 in NPC SUNE1 and 5-8F cells. (c) From mock and pcDNA3.1-C-JUNtransfected SUNE1 cells was immunoprecipitated with anti-C-JUN and a normal rabbit IgG. The AP-1 binding sites on the immunoprecipitated DNA was determined by quantitative RT-PCR. Amplification of input chromatin (input) before immunoprecipitation was served as positive controls for chromatin extraction and PCR amplification. Chromatin immunoprecipitation using a non-specific antibody (normal human IgG) served as negative controls. QPCR analysis indicated that C-Jun could bind more miR-184 promoter region than that in control group in NPC SUNE1 cells. (d) Suppressing the expression of PI3K by its specific inhibitor Ly294002 (50 nM) reduced pPI3K, pAKT and C-JUN expression in NPC SUNE1 and 5-8F cells, but did not induce AKT expression change. (e) Knocking down JNK1 suppressed the expression of C-Jun in NPC SUNE1 and 5-8F cells. (f) Specific inhibition of JNK1 by siRNA decreased the expression of JNK and C-Jun in NPC cells. Data are presented as mean \pm S.D. of three independent experiments $\left({ }^{*} P<0.05\right)$

the cytoplasm and nuclei of malignant epithelial cells and noncancerous tissues but predominantly expressed in cytoplasm by immunohistochemistry. Furthermore, we also found that attenuated PDCD4 expression was negatively associated with $\mathrm{T}$ classification, $\mathrm{N}$ classification and clinical stages of NPC patients. Furthermore, we also observed that lost PDCD4 protein expression in NPC was positively correlated with patient's overall survival. Patients with lower expression of PDCD4 protein had a shorter overall survival time. Multivariate analyses showed that decreased expression of PDCD4 protein was a significant independent predictor of poor prognosis for NPC patients. The results mentioned above suggested PDCD4 as a significant suppressor in NPC, which is similar to other reports of PDCD4 in some tumors. ${ }^{19-22}$

Subsequently, we presented the evidence that PDCD4 was taken a tumor suppressor participating in NPC pathogenesis.
We found that PDCD4-mediated growth suppression attributed to cell cycle transition obstacle by repressing the expression of cell cycle factors, including C-MYC, CCND1, $\mathrm{pRb}$ and E2F1, and transcription factor C-Jun, and the elevation of cell apoptosis level by inducing the cleavage of CASP9, 3, 6, 7 and PARP.

In previous studies, PDCD4-mediated miRNA regulation in tumor cell growth and apoptosis was rarely reported. In this investigation, miR-184 was shown to be significantly downexpressed in NPC cells of knocking down PDCD4 expression compared to scramble NPC cells by miRNA array and qPCR examinations. Dysregulation of miR-184 was preliminarily reported as a potential oncomir promoting cell proliferation and blocking cell apoptosis in tongue carcinoma. ${ }^{23}$ In subsequent studies, miR-184 was shown as a tumor suppressor involved in suppressing cell survival and growth by targeting AKT2 in neuroblastoma cell. ${ }^{24}$ In our study, 
miR-184, identified as a potential tumor suppressor, repressed cell growth by modulating C-MYC-controlled cell cycle transition and BCL2-suppressed mitochondrion apoptosis, which was consistent with the role of PDCD4 in NPC. However, miR-184 mimics did not control the expression of PDCD4, which hinted that miR-184 was a downstream regulator of PDCD4. Subsequently, we investigated miR184-regulated genes possibly involved in cell cycle and apoptosis pathways. Excitingly, C-MYC coding region and BCL-2 3'UTR were predicted as miR-184-targeted region by bioinformatics assay. Our experiments further validated this speculation that miR-184 directly targeted C-MYC and BCL-2 expression. These results demonstrated that miR-184 participated in PDCD4-mediated suppression of cell growth and survival in NPC.

To understand the molecular mechanism of PDCD4-modulated miR-184, we first employed bioinformatics assay to find that there were binding sites of AP1 (C-JUN), an oncogenic transcription factor also regulated by PI3K/AKT and JNK signal, ${ }^{25,26}$ a classic pathway stimulating tumor pathogenesis, ${ }^{27-29}$ in putative promoter region of miR-184. We speculated that PDCD4 might participate in modulating the expression of miR-184 through PI3K/AKT and JNK/C-JUN pathway. Our results demonstrated that PDCD4 inactivated PI3K/AKT and JNK-mediated activation of C-JUN, which reduced the repression of miR-184 promoter and stimulated miR-184 expression in NPC. These results were in line with our expectation.

In addition to the effects of miR-184-mediated suppression of PDCD4 in cell proliferation and survival in NPC, we observed that the expression pattern of miR-184 was analogous to that of PDCD4 in NPC. Furthermore, we also found that miR-184 was positively correlated with PDCD4 mRNA levels, suggesting that miR-184 might have important roles in NPC tumorigenesis of PDCD4 dysfunction.

As summarized in Supplementary Figure S9, this study provided evidence that PDCD4 was downregulated and its reduced expression in cellular cytoplasm facilitated the development and poor prognosis in NPC. Furthermore, we also identified the abnormal pathways of PDCD4 in suppressing cell growth and survival by miR-184-mediated direct targeting of BCL2 and C-MYC via PI3K/AKT and JNK/C-Jun pathway in NPC.

\section{Materials and Methods}

Cell culture. Eight NPC cell lines 5-8F, 6-10B, CNE1, CNE2, C666-1, HONE1, HNE1 and SUNE1 were maintained in RPMI 1640 medium supplemented with $10 \%$ newborn calf serum (NBCS) (PAA Laboratories, Inc., Pasching, Austria). All the cell lines were incubated in a humidified chamber with $5 \% \mathrm{CO}_{2}$ at $37^{\circ} \mathrm{C}$.

Collection of primary NPC and noncancerous nasopharynx specimens and ethics statement. Sixty-three fresh NPC tissues, 20 fresh nasopharynx tissuses, 190 paraffin-embedded undifferentiated NPC specimens and 121 paraffin-embedded noncancerous nasopharynx specimens (including 76 normal epithelium, 22 squamous epithelium and 23 atypical hyperplasia epithelium) were obtained at the time of diagnosis before any therapy from People's Hospital in Zhongshan City (Guangdong, China). In these 190 NPC cases with clinical and prognosis information, there were 128 male and 62 female patients with age ranging from 19 to 80 years (median, 49.12 years). The clinical follow-up time of patients ranged from 4 to 126 months. The clinical processes were approved by the Ethics Committees of People's Hospital of Zhongshan City. The patients provided informed consents. The pathologic stage of all specimens was confirmed according to the 1997 NPC staging system of the WHO.
Analysis of microarray data. Microarray data set (GEO accession number: GSE2370) from five representative EBV-negative NPC cell lines: TW01, TW03, TW04, TW06 and CGBM1 and normal nasal mucosal epithelial submitted by Lee was retrieved from the GEO database and differentially expressed genes were screened with a selection criteria based on our previous investigation. $^{9}$

Construction of pGC-FU-PDCD4-GFP vector and lentivirus infection. PDCD4 cDNA was cloned into an Agel-digested pGC-FU-GFP lentivirus vector (Genechem Incorporation, Shanghai, China). The resulting lentivirus vector together with pHelper1 and pHelper2 vectors were cotransfected into 293FT cells for $84 \mathrm{~h}$ using lipofectamine 2000 (Invitrogen, Carlsbad, CA, USA) to generate lentiviral stock. The lentiviral particles were used to infect $5-8 \mathrm{~F}$ cells with high ability of tumorigenesis and metastasis, ${ }^{30}$ and colonies with GFP expression were selected to expand culture for further investigation.

Establishment of NPC HONE1 and SUNE1 cells with stable expression of PDCD4 shRNA. We selected two shRNA sequences in 1290 and 1605 of PDCD4 mRNA sequence (Supplementary Table S1) for targeting PDCD4 gene using the BLOCK-It RNAi Designer (Invitrogen). The preparation of lentiviral vectors expressing human PDCD4 shRNA was performed using the pLVTHM-GFP lentiviral RNAi expression system. ${ }^{31}$ NPC HONE1 and SUNE1 cells were infected with lentiviral particles containing specific or negative control vectors, and the polyclonal cells with GFP signals were selected for further experiments using FACS flow cytometer assay.

Western blot analysis. Cells were lysed in RIPA buffer $(50 \mathrm{mM}$ Tris- $\mathrm{HCl}$, pH8.0; $1 \mathrm{mM}$ ethylenediaminetetraacetic acid, pH 8.0; $5 \mathrm{mM} \mathrm{DTT;} 2 \%$ sodium dodecyl sulfate (SDS)), and protein concentration was determined using BCA assay (Beyotime, Beijing, China). Total protein $(30 \mu \mathrm{g}$ ) was resolved using a $10 \%$ SDS-polyacrylamide gel electrophoresis gel and electro-transferred to polyvinylidene fluoride membranes (Invitrogen). Membranes were blocked with $5 \%$ non-fat dry milk (for phosphorylation antibody, adding BSA) in Tris-buffered saline ( $\mathrm{pH}$ 7.5), followed by immunobloting overnight at $4^{\circ} \mathrm{C}$ with anti-PDCD4 antibody (1:1000; Cell Signaling Technology, Danvers, MA, USA), anti- $\beta$-actin, GAPDH, CCND1, CDK4, p21, E2F1 and p15 antibody (1: 400; Santa Cruz Biotechnology, Santa Cruz, CA, USA), anti-pRb (Ser780), C-MYC, C-JUN, AKT, pAKT (Ser473), PI3K, pPI3K (Tyr458), cleaved-caspase 3, 6, 7, 9, PARP (1:1000; Cell Signaling Technology), BCL2 (1:1000; Abcam, Cambridge, UK), JNK and p27 (1:1000; Epitomics, Burlingame, CA, USA). A horseradish peroxidase-conjugated antirabbit and anti-mouse IgG antibody was used as the secondary antibody (Zhongshan, Beijing, China). Signals were detected using enhanced chemiluminescence reagents (Pierce, Rockford, IL, USA).

RNA isolation, reverse transcription, and qRT-PCR. RNA was extracted from the NPC cell lines, NPC tissues and normal nasopharynx tissues using Trizol (Takara, Shiga, Japan). For miR-184 qRT-PCR, RNA was transcribed into CDNA and amplified with specific sense primers (Supplementary Table S2), and general antisense primer was supplied by manufacturer using miRNA PrimeScript RT Enzyme Mix kit according to the manufacturer's instructions (Ambion, Life Technologies, Carlsbad, CA, USA). For PDCD4 and C-JUN qRTPCR, RNA was transcribed into CDNA and amplified with specific sense/antisense primer (Supplementary Table S3). The assays were performed in accordance with manufacturer's instructions (Takara). The PCR reaction for each gene was repeated three times. miRNA and mRNA expression were normalized to U6 (Supplementary Table S2) and ARF5 (Supplementary Table S3), respectively, using the $2^{-\Delta \Delta \mathrm{Ct}}$ method.

Immunohistochemistry and evaluation of staining. Paraffin sections $(4 \mu \mathrm{m})$ from samples were deparaffinized in $100 \%$ xylene and rehydrated in descending ethanol series and water according to standard protocols. Heatinduced antigen retrieval was performed in $10 \mathrm{mM}$ citrate buffer for $2 \mathrm{~min}$ at $100^{\circ} \mathrm{C}$. Endogenous peroxidase activity and nonspecific antigen were blocked with peroxidase-blocking reagent containing $3 \%$ hydrogen peroxide and serum, followed by incubation with rabbit anti-human PDCD4 antibody $(1: 100$, Cell Signaling Technology) for overnight at $4^{\circ} \mathrm{C}$. After washing, the sections were incubated with biotin-labeled rabbit anti-goat antibody for $10 \mathrm{~min}$ at room temperature, and subsequently were incubated with streptavidin-conjugated horseradish peroxidase (Maixin, Fuzhou, China). The peroxidase reaction was 
developed using 3,3-diaminobenzidine chromogen solution in DAB buffer substrate. Sections were visualized with $D A B$ and counterstained with hematoxylin, mounted in neutral gum and analyzed using a bright field microscope. The immunohistochemically stained tissue sections were reviewed and scored separately by two pathologists blinded to the clinical parameters. For cytoplasmic staining, the score was evaluated according to the sum of cytoplasm staining intensity and the percentage of positive staining areas of cells. The staining intensity was scored as previously described $(0-3)^{10}$ and the percentage of positive staining areas of cells was defined as a scale of $0-3(0:<10 \%, 1$ : $10-25 \%, 2: 26-75 \%$ and $3: \geq 76 \%$ ). For statistical analysis, a final staining score of $0-4$ and $5-6$ in cytoplasm was considered to be low and high expression, respectively.

Cell cycle analysis. For cell cycle analysis, cells were seeded on $10-\mathrm{cm}$ diameter plates in RPMI 1640 containing $10 \%$ NBCS. After incubation for $48 \mathrm{~h}$, a total of $5 \times 10^{6}$ cells were harvested, rinsed with cold PBS and fixed with $70 \%$ ice-cold ethanol for $48 \mathrm{~h}$ at $4^{\circ} \mathrm{C}$. Fixed cells were rinsed with cold PBS followed by incubation with PBS containing $10 \mu \mathrm{g} / \mathrm{ml}$ propidium iodide and $0.5 \mathrm{mg} / \mathrm{ml}$ RNase A for $30 \mathrm{~min}$ at $37^{\circ} \mathrm{C}$. The DNA content of labeled cells was acquired using FACS cytometry assay (BD Biosciences, Franklin Lakes, NJ, USA). Each experiment was performed in triplicate.

Apoptosis assays. Apoptosis was demonstrated by Annexin V-Cy5 Apoptosis Detection Kit K103-25 (Biovision Corporation, San Francisco, CA, USA). Briefly, $1-5 \times 10^{5}$ cells were collected, washed twice in cold PBS and resuspended in $500 \mu \mathrm{l}$ binding buffer. The suspension cells were stained with $5 \mu \mathrm{l}$ Annexin V-Cy5 and incubated for $5 \mathrm{~min}$ at room temperature in the dark. Apoptotic cells were assessed using FACS (BD Biosciences). Experiments were performed in multiples to qualify apoptosis by phosphatidylserine externalization.

Cell proliferation analysis. Cell proliferation was analyzed using MTT assay. Briefly, $1 \times 10^{3}$ cells were seeded into a 96-well plate with quadruplicate repeat for each condition. For shRNA-PDCD4 and PGC-FU-PDCD4, the cells were incubated for 1, 2, 3, 4, 5, 6 and 7 days. For miR-184 mimics, the cells were incubated for 1, 2 and 3 days. Twenty microliters of MTT ( $5 \mathrm{mg} / \mathrm{ml}$ ) (Sigma, St Louis, MO, USA) was added to each well and incubated for $4 \mathrm{~h}$. At the end of incubation, the supernatants were removed and $150 \mu \mathrm{l}$ of DMSO (Sigma) was added to each well. The absorbance value (OD) of each well was measured at $490 \mathrm{~nm}$. Experiments were performed three times.

Colony formation assay. Cells were plated in six-well culture plates at 100 cells/well. Each cell group had two wells. After incubation for 12 days at $37^{\circ} \mathrm{C}$, cells were washed twice with PBS and stained with the Giemsa solution (SigmaAldrich Corporation, St. Louis, MO, USA). The number of colonies containing $\geq 50$ cells was counted under a microscope. The colony formation efficiency was calculated as (number of colonies/number of cells inoculated) $\times 100 \%$.

In vivo tumorigenesis in nude mice. A total of $1 \times 10^{6}$ logarithmically grown 5-8F cells transfected with pGC-FU-GFP-PDCD4 (clone 1 and 2) and the control pGC-FU-GFP vector (scramble) in $0.1 \mathrm{ml}$ RPMI 1640 medium were subcutaneously injected into the left flank of 4-6-week-old male BALB/c nu/nu mice. The mice were maintained in a barrier facility on HEPA-filtered racks. The animals were fed with an autoclaved laboratory rodent diet. All animal studies were conducted in accordance with the principles and procedures outlined in Southern Medical University Guide for the Care and Use of Animals under assurance number SCXK (Guangdong) 2008-0002. After 19 days the mice were killed and tumor tissues were excised and weighted.

miRNA array for shPDCD4. We used a custom-made, two-channel oligo array (CCDTM-miRNA850-V4px) from NIH including 713 human, mammalian and viral mature antisense miRs plus two internal controls with seven serial dilutions, ${ }^{16}$ which were endowed by Professor Wang E and Marincola FM from National Institutes of Health, Bethesda, USA. Total RNA samples $(6-7 \mu \mathrm{g})$ isolated from each shPDCD4 and its control NPC SUNE1 cells were labeled by Cy5-dCTP and Cy3-dCTP, respectively, in a reverse transcription reaction. The balanced mixture of Cy5- and Cy3-labeled targets was cohybridized against miRNA chips. The experiments were repeated four times and the data were analyzed using and BRB ArrayTools V3.7.2 software (National Cancer Institute, Bethesda, MD, USA).
miRNA target validation. BCL2 and C-MYC were predicted to be directly regulated targets of miR-184 by RNAhybrid (The BiBiServ, Bielefeld, Germany) and miRwalk softwares (University of Heidelberg, Mannheim, Germany). A 420-bp fragment of BCL2 3'UTR or 309-bp fragment of C-MYC CDS (coding sequence) amplified by PCR primers (Supplementary Table S4) was cloned into psiCHECK-2 vectors (named wt). Site-directed mutagenesis of the miR-184 binding site in BCL2 3'UTR and C-MYC CDS were performed using GeneTailor Site-Directed Mutagenesis System (Invitrogen; named mt). For reporter assays, wt or mt vector and the control vector psiCHECK-2 vector were cotransfected into SUNE1 cells with miR-184 mimics or inhibitor. Luciferase activity was measured at $48 \mathrm{~h}$ after transfection using the Dual-Luciferase Reporter Assay System (Promega Corporation, Madison, WI , USA).

Transient transfection with siRNAs or miR-184 mimics/inhibitor or PI3K inhibitor Ly294002. siRNA for C-JUN and JNK1 (Supplementary Tables S5 and 6) (RiboBio Inc., Guangzhou, China) or miR-184 mimics and its inhibitor were designed and synthesized (GenePharma Co., Ltd, Shanghai, China). The sequence of each miRNA and their controls are shown in Supplementary Table S7. PI3K inhibitor Ly294002 was bought from Sigma. Twenty-four hours before transfection, NPC cells $5-8 \mathrm{~F}$ and SUNE1 were plated onto a 6- or 96-well plate (Nest, Biotech, China) at 30-50\% confluence. siRNA or miRNAs were then transfected at a working concentration of $100 \mathrm{nM}$ using TurboFect siRNA Transfection Reagent (Fermentas, Vilnius, Lithuania) according to the manufacturer's protocol. Cells were collected after $48-72 \mathrm{~h}$ for the further experiments.

Chromatin immunoprecipitation assay. DNA-protein complexes were immunoprecipitated from SUNE1 cells by using the Chromatin Immunoprecipitation Kit (Millipore, Billerica, MA, USA) according to the manufacturer's protocol with the following polyclonal antibodies: anti-C-JUN and normal mouse IgG (Millipore), the latter served as a control for nonspecific DNA binding. The precipitated DNA was subjected to $\mathrm{gPCR}$ analysis using specific primers. The primers (Supplementary Table S8) were utilized to amplify across the miR-184 promoter region.

Statistical analysis. All data were analyzed for statistical significance using SPSS 13.0 software (SPSS, Chicago, IL, USA). The chi-square test was applied to the examination of relationship between PDCD4 expression levels and clinicopathological characteristics. Survival analysis was performed using KaplanMeier method. Multivariate Cox proportional hazards method was used for analyzing the relationship between the variables and patient's survival time. The relationship between PDCD4 and miR-184 expression was explored by Pearson test. Two-tailed Student's $t$ test was used for comparisons of two independent groups. One-way ANOVA was used to determine the differences between groups for all in vitro analyses. A P-value of $<0.05$ was considered statistically significant.

\section{Conflict of Interest}

The authors declare no conflict of interest.

Acknowledgements. This study was supported by National Nature Science Fund of China (No. 81372184, No. 30872856 and No. 81272268) (http:// www.nsfc.gov.cn), Nature Science Fund of Guangdong Province No. S2013010014724), new star plan of Pearl River Science and Technology from Guangzhou City (No. 2011J2200009) and Guangdong Province Breeding Project Plan (LYM11102).

1. Goke A, Goke R, Knolle A, Trusheim H, Schmidt H, Wilmen A et al. DUG is a novel homologue of translation initiation factor $4 \mathrm{G}$ that binds elF4A. Biochem Biophys Res Commun 2002; 297: 78-82.

2. Ponting CP. Novel elF4G domain homologues linking mRNA translation with nonsensemediated mRNA decay. Trends Biochem Sci 2000; 25: 423-426.

3. Bohm M, Sawicka K, Siebrasse JP, Brehmer-Fastnacht A, Peters R, Klempnauer KH. The transformation suppressor protein Pdcd4 shuttles between nucleus and cytoplasm and binds RNA. Oncogene 2003; 22: 4905-4910.

4. Reis PP, Tomenson M, Cervigne NK, Machado J, Jurisica I, Pintilie M et al. Programmed cell death 4 loss increases tumor cell invasion and is regulated by miR-21 in oral squamous cell carcinoma. Mol Cancer 2010; 9: 238. 
5. Bitomsky N, Bohm M, Klempnauer KH. Transformation suppressor protein Pdcd4 interferes with JNK-mediated phosphorylation of c-Jun and recruitment of the coactivator p300 by c-Jun. Oncogene 2004; 23: 7484-7493.

6. Bitomsky N, Wethkamp N, Marikkannu R, Klempnauer KH. siRNA-mediated knockdown of Pdcd4 expression causes upregulation of p21(Waf1/Cip1) expression. Oncogene 2008; 27: 4820-4829.

7. Ozpolat B, Akar U, Steiner M, Zorrilla-Calancha I, Tirado-Gomez M, Colburn N et al. Programmed cell death-4 tumor suppressor protein contributes to retinoic acid-induced terminal granulocytic differentiation of human myeloid leukemia cells. Mol Cancer Res 2007; 5: 95-108.

8. Wei N, Liu SS, Chan KK, Ngan HY. Tumour suppressive function and modulation of programmed cell death 4 (PDCD4) in ovarian cancer. PLoS One 2012; 7: e30311.

9. Fang W, Li X, Jiang Q, Liu Z, Yang H, Wang S et al. Transcriptional patterns, biomarkers and pathways characterizing nasopharyngeal carcinoma of Southern China. J Transl Med 2008; 6: 32.

10. Liu Z, Li X, He X, Jiang Q, Xie S, Yu X et al. Decreased expression of updated NESG1 in nasopharyngeal carcinoma: its potential role and preliminarily functional mechanism. Int J Cancer 2011; 128: 2562-2571.

11. Yu X, Zhen $Y$, Yang $H$, Wang $H$, Zhou $Y$, Wang $E$ et al. Loss of connective tissue growth factor as an unfavorable prognosis factor activates miR-18b by PI3K/AKT/C-Jun and C-Myc and promotes cell growth in nasopharyngeal carcinoma. Cell Death Dis 2013; 4: e634.

12. Lu J, He ML, Wang L, Chen Y, Liu X, Dong $Q$ et al. MiR-26a inhibits cell growth and tumorigenesis of nasopharyngeal carcinoma through repression of EZH2. Cancer Res 2011; 71: 225-233.

13. Alajez NM, Shi W, Hui AB, Bruce J, Lenarduzzi M, Ito E et al. Enhancer of Zeste homolog 2 $(E Z H 2)$ is overexpressed in recurrent nasopharyngeal carcinoma and is regulated by miR-26a, miR-101, and miR-98. Cell Death Dis 2010; 1: e85.

14. Lee YC, Hwang YC, Chen KC, Lin YS, Huang DY, Huang TW et al. Effect of Epstein-Barr virus infection on global gene expression in nasopharyngeal carcinoma. Funct Integr Genomics 2007; 7: 79-93.

15. Wang S, Fang W. Increased expression of hepatoma-derived growth factor correlates with poor prognosis in human nasopharyngeal carcinoma. Histopathology 2011; 58: 217-224.

16. Wang WQ, Zhang $H$, Wang HB, Sun YG, Peng ZH, Zhou G et al. Programmed cell death 4 (PDCD4) enhances the sensitivity of gastric cancer cells to TRAlL-induced apoptosis by inhibiting the PI3K/Akt signaling pathway. Mol Diagn Ther 2010; 14 155-161.

17. Subedi A, Kim MJ, Nepal S, Lee ES, Kim JA, Sohn DH et al. Globula adiponectin modulates expression of programmed cell death 4 and miR-21 in RAW 264.7 macrophages through the MAPK/NF- $\kappa$ B pathway. FEBS Lett 2013; 587: 1556-1561.

18. Luo H, Yang Y, Duan J, Wu P, Jiang Q, Xu C. PTEN-regulated AKT/FoxO3a/Bim signaling contributes to reactive oxygen species-mediated apoptosis in selenite-treated colorectal cancer cells. Cell Death Dis 2013; 4: e481.
19. Mudduluru G, Medved F, Grobholz R, Jost C, Gruber A, Leupold JH et al. Loss of programmed cell death 4 expression marks adenoma-carcinoma transition, correlates inversely with phosphorylated protein kinase $\mathrm{B}$, and is an independent prognostic factor in resected colorectal cancer. Cancer 2007; 110: 1697-1707.

20. Wang Q, Sun Z, Yang HS. Downregulation of tumor suppressor Pdcd4 promotes invasion and activates both beta-catenin/Tcf and AP-1-dependent transcription in colon carcinoma cells. Oncogene 2008; 27: 1527-1535.

21. Cao Z, Yoon JH, Nam SW, Lee JY, Park WS. PDCD4 expression inversely correlated with miR-21 levels in gastric cancers. J Cancer Res Clin Oncol 2012; 138: 611-619.

22. Li X, Xin S, Yang D, Li X, He Z, Che X et al. Down-regulation of PDCD4 expression is an independent predictor of poor prognosis in human renal cell carcinoma patients. J Cancer Res Clin Oncol 2012; 138: 529-535.

23. Wong TS, Liu XB, Wong BY, Ng RW, Yuen AP, Wei WI. Mature miR-184 as potential oncogenic microRNA of squamous cell carcinoma of tongue. Clin Cancer Res 2008; 14: 2588-2592.

24. Foley NH, Bray IM, Tivnan A, Bryan K, Murphy DM, Buckley PG et al. MicroRNA-184 inhibits neuroblastoma cell survival through targeting the serine/threonine kinase AKT2. Mol Cancer 2010; 9: 83

25. Ivanov VN, Krasilnikov M, Ronai Z. Regulation of Fas expression by STAT3 and C-Jun is mediated by phosphatidylinositol 3-kinase-AKT signaling. J Biol Chem 2002; 277: 4932-4944.

26. Zhang JY, Adams AE, Ridky TW, Tao S, Khavari PA. Tumor necrosis factor receptor $1 / \mathrm{c}$ Jun-NH2-kinase signaling promotes human neoplasia. Cancer Res 2007; 67: 3827-3834.

27. Yang $\mathrm{CH}$, Kao YH, Huang KS, Wang CY, Lin LW. Cordyceps militaris and mycelial fermentation induced apoptosis and autophagy of human glioblastoma cells. Cell Death Dis 2012; 3: e431.

28. Gao N, Budhraja A, Cheng S, Liu EH, Chen J, Yang Z et al. Phenethyl isothiocyanate exhibits antileukemic activity in vitro and in vivo by inactivation of Akt and activation of JNK pathways. Cell Death Dis 2011; 2: e140.

29. Vaseva AV, Yallowitz AR, Marchenko ND, Xu S, Moll UM. Blockade of Hsp90 by 17AAG antagonizes MDMX and synergizes with Nutlin to induce p53-mediated apoptosis in solid tumors. Cell Death Dis 2011; 2: e156.

30. Song LB, Yan J, Jian SW, Zhang L, Li MZ, Li D et al. Molecular mechanisms of tumorgenesis and metastasis in nasopharyngeal carcinoma cell sublines. Ai Zheng 2002; 21: $158-162$

31. Liu Z, Luo W, Zhou Y, Zhen Y, Yang H, Yu X et al. Potential tumor suppressor NESG1 as an unfavorable prognosis factor in nasopharyngeal carcinoma. PLoS One 2011; 6: e27887.

(c) (i) () $\odot$ Cell Death and Disease is an open-access journal cc) published by Nature Publishing Group. This work is licensed under a Creative Commons Attribution-NonCommercialNoDerivs 3.0 Unported License. To view a copy of this license, visit http://creativecommons.org/licenses/by-nc-nd/3.0/

Supplementary Information accompanies this paper on Cell Death and Disease website (http://www.nature.com/cddis) 